\title{
PARTITIONING THE POSITIVE INTEGERS WITH HIGHER ORDER RECURRENCES
}

\section{CLARK KIMBERLING}

University of Evansville

1800 Lincoln Avenue, Evansville, IN 47722

(Recelved June 17, 1990 and in revised form January 25, 1991)

ABSTRACT. Associated with any irrational number $\alpha>1$ and the function $g(n)=\left[\alpha n+\frac{1}{2}\right]$ is an array $\{s(i, j)\}$ of positive integers defined inductively as follows: $s(1,1)=1, s(1, j)=g(s(1, j-1))$ for all $j \geq 2, s(i, 1)=$ the least positive integer not among $s(h, j)$ for $h \leq i-1$ for $i \geq 2$, and $s(i, j)=g(s(i, j-1))$ for $j \geq 2$. This work considers algebraic integers $\alpha$ of degree $\geq 3$ for which the rows of the array $s(i, j)$ partition the set of positive integers. Such an array is called a Stolarsky array. A typical result is the following (Corollary 2): if $\alpha$ is the positive root of $x^{k}-x^{k-1}-\ldots-x-1$ for $k \geq 3$, then $s(i, j)$ is a Stolarsky array.

KEY WORDS AND PHRASES. Stolarsky array, linear recurrence sequence, nearly arithmetic sequence, nearly geometric sequence.

1980 AMS SUBJECT CLASSIFICATION CODE. 11 B37 .

\section{INTRODUCTION.}

It is possible to partition the set $Z^{+}$of positive integers as an infinite set of sequences all obeying a common linear recurrence relation. Perhaps the first such array was introduced in 1977 by Stolarsky [1]. The first row of Stolarsky's array is the sequence of Fibonacci numbers, $1,2,3,5,8,13, \ldots$ as seen

in Table 1. This row and all subsequent rows obey the recurrence $s_{\jmath}=s_{j-1}+s_{j-2}$ for all $j \geq 3$. Explicitly, each row after the first begins with the least positive integer not in any previous row, and all terms following the first term of a row are then given by the simple nonlinear recurrence $s_{\jmath}=\left[\alpha s_{\jmath-1}+\frac{1}{2}\right] ;$ that is, the integer nearest $\alpha s_{\jmath-1}$, where $\alpha$ is the positive root of the characteristic polynomial $x^{2}-x-1$ of the linear recurrence $s_{\jmath}=s_{\jmath-1}+s_{\jmath-2}$.

The purpose of this paper is to determine other linear recurrence relations, notably of order $\geq 3$, for which the first-order nonlinear recurrence $s_{\jmath}=\left[\alpha s_{\jmath-1}+\frac{1}{2}\right]$, for suitable $\alpha$, generates an array that partitions $Z^{+}$in the manner of generation of Stolarsky's array. Burke and Bergum [2], Butcher [3], Gbur [4], Hendy [5], Kimberling [6], Morrison [7], and Stolarksy [1] deal with Stolarsky's original array or other arrays with row sequences linearly recurrent with order 2 . The results presented here for higher order recurrences are believed to be new.

\section{DEFINITIONS AND AN EXAMPLE.}

An array $s(i, j), i \geq 1, j \geq 1$, of positive integers is a Stolarsky array [specifically, a $\left(c_{k-1}, c_{k-2}\right.$, $\left.\ldots, c_{0}\right)$ Stolarsky array] if 
(i) every positive integer occurs exactly once in the array, and

(ii) there exist integers $c_{k-1}, c_{k-2}, \ldots, c_{0}$, where $c_{0} \neq 0$ and $k \geq 2$, such that

$$
s(i, j)=c_{k-1} s(i, j-1)+c_{k-2} s(i, j-2)+\ldots+c_{0} s(i . j-n)
$$

for all $j \geq k+1$ for all $i \geq 1$.

For a given Stolarsky array, if $k$ is the least positive integer for which (2.1) holds for some choice of integers $c_{k-1}, c_{k-2}, \ldots, c_{0}$, where $c_{0} \neq 0$, then these integers are uniquely determined, and identity (2.1) is the recurrence of the array. The array has order $k$, and the polynomial $x_{k}-c_{k-1} x^{k-1}-$ $c_{k-2} x^{k-2}-\ldots-c_{1} x-c_{0}$ is the characteristic polynomial of the array.

$\begin{array}{rrrrrrrr}1 & 2 & 3 & 5 & 8 & 13 & 21 & \ldots \\ 4 & 6 & 10 & 16 & 26 & 42 & 68 & \ldots \\ 7 & 11 & 18 & 29 & 47 & 76 & 123 & \ldots \\ 9 & 15 & 24 & 39 & 63 & 102 & 165 & \ldots \\ 12 & 19 & 31 & 50 & 81 & 131 & 212 & \ldots \\ 14 & 23 & 37 & 60 & 97 & 157 & 254 & \ldots \\ 17 & 28 & 45 & 73 & 118 & 191 & 309 & \ldots\end{array}$

Table 1: The First Seven Rows of Stolarsky's Array

As an example, consider the array in Table 2, found by using the dominant real root $\alpha=$ 3.62736508471183 of $x^{3}-3 x^{2}-2 x-1$ (in Table 3).

In Table 2 , it is easy to verify that the numbers $s(i, j)$ in Row $i$, for each $i \geq 1$, satisfy the recurrence

$$
s(i, j)=3 s(i, j-1)+2 s(i, j-2)+s(i, j-3)
$$

for all $j \geq 4$. Now $\alpha$ exceeds 1 and (by Corollary 3) $s(i, j)=\left[\alpha s(i, j-1)+\frac{1}{2}\right]$ for all $j \geq 2$ for all $i \geq 1$. Here again note that $s(1,1)=1$ by definition. It follows that each positive number appears once and only once in the array, as $s(i, 1)$ for $i \geq 2$ is, by construction, the least positive integer not among $s(h, j)$ for $1<h \leq i-1$ and $j \geq 1$. Therefore, the array is a third-order Stolarsky array.

$\begin{array}{rrrrrrrrl}1 & 4 & 15 & 54 & 196 & 711 & 2579 & 9355 & \ldots \\ 2 & 7 & 25 & 91 & 330 & 1197 & 4342 & 15750 & \ldots \\ 3 & 11 & 40 & 145 & 526 & 1908 & 6921 & 25105 & \ldots \\ 5 & 18 & 65 & 236 & 856 & 3105 & 11263 & 40855 & \ldots \\ 6 & 22 & 80 & 290 & 1052 & 3816 & 13842 & 50210 & \ldots \\ 8 & 29 & 105 & 381 & 1382 & 5013 & 18184 & 65960 & \ldots\end{array}$

Table 2: A Third-order Stolarsky Array

Our main objective can now be stated as follows: to determine polynomials

$$
f(x)=x^{k}-c_{k-1} x^{k-1}-\ldots-c_{1} x-c_{0}
$$


for which the fornula $\left[\alpha n+\frac{1}{2}\right]$ generates a Stolarsky array in the manner of the above example.

\section{CONDITIONS FOR GENERATING STOLARSKY ARRAYS.}

LEMMA 1: If $\alpha>1$ and $m$ and $n$ are positive integers satisfying $m<n$, then $\left[\alpha m+\frac{1}{2}\right]<\left[\alpha n+\frac{1}{2}\right]$.

PROOF: If $m \leq n-1$ then $\alpha m \leq \alpha n-\alpha<\alpha n-1$, so that

$$
\left[\alpha m+\frac{1}{2}\right] \leq\left[\alpha n-1+\frac{1}{2}\right]<\left[\alpha n+\frac{1}{2}\right]
$$

Lemma 2: Suppose the polynomial (2) has a dominant real root $\alpha>1$. For arbitrary positive integer $n$, let $g(n)=\left[\alpha n+\frac{1}{2}\right], g^{2}(n)=g(g(n)), \ldots, g^{k}(n)=g\left(g^{k-1}(n)\right), \ldots$ If

$$
g^{k+m}(n)=c_{k-1} g^{k+m-1}(n)+\ldots+c_{1} g^{m+1}(n)+c_{0} g^{m}(n),
$$

where $g^{0}(n)=n$, holds for $m=0$ and all $n \geq 1$, then (3.1) holds for all $m \geq 0$ for all $n \geq 1$.

Proof:

$$
\begin{aligned}
g^{k+m}(n) & =g^{k}\left(g^{m}(n)\right) \\
& =c_{k-1} g^{k-1}\left(g^{m}(n)\right)+\ldots+c_{1} g\left(g^{m}(n)\right)+c_{0} g^{m}(n) \\
& =c_{k-1} g^{k+m-1}(n)+\ldots+c_{1} g^{m+1}(n)+c_{0} g^{m}(n)
\end{aligned}
$$

TheOREM 1: Let $r_{1}=\left(\left(\alpha n+\frac{1}{2}\right)\right)$ be the fractional part of $g(n)$ in Lemma 2. That is, $r_{1}=$ $\alpha n+\frac{1}{2}-\left[\alpha n+\frac{1}{2}\right]$. Let $r_{2}=\left(\left(\alpha g(n)+\frac{1}{2}\right)\right), \ldots, r_{k}=\left(\left(\alpha g^{k-1}(n)+\frac{1}{2}\right)\right)$. Let

$$
\begin{aligned}
M= & \frac{1}{2(\alpha-1)}\left(c_{0}+c_{1}+\ldots+c_{k-1}-1\right)-\frac{r_{1} c_{0}}{\alpha}-\frac{r_{2}\left(c_{0}+c_{1} \alpha\right)}{\alpha^{2}} \\
& -\frac{r_{3}\left(c_{0}+c_{1} \alpha+c_{2} \alpha^{2}\right)}{\alpha^{3}}-\ldots-\frac{r_{k-1}\left(c_{0}+c_{1} \alpha+\ldots+c_{k-2} \alpha^{k-2}\right)}{\alpha^{k-1}}-r_{k} .
\end{aligned}
$$

Let $s(1, j)=\left[\alpha j+\frac{1}{2}\right]$ for $j \geq 1$ and $s(1,1)=1$. Define $s(i, j)$ inductively by letting $s(i, 1)$ be the least positive integer not among $s(h, j)$ for $1<h \leq i-1$ and $j \geq 1$, and $s(i, j)=\left[\alpha s(i, j-1)+\frac{1}{2}\right]$ for $j \geq 2$. Then $\{s(i, j)\}$ is a Stolarsky array if and only if $|M|<1$.

Before proving Theorem 1, we use the notation introduced there to establish a lemma:

LEMMA 3: $g^{i}(n)=\alpha^{i} n+\left(\frac{1}{2}\right) \frac{\alpha^{i}-1}{\alpha-1}-\sum_{j=1}^{i} r, \alpha^{i-j}$ for $i \geq 1$.

Proof: $g(n)=\alpha n+\frac{1}{2}-r_{1}$, as asserted for $i=1$. The identity follows by induction on $i$.

Proof of Theorem 1: In view of Lemmas 1 and 2, it suffices to show that the inequality $|M|<1$ is equivalent to identity (3.1) for $m=0$ and all $n \geq 1$.

$$
\begin{aligned}
g^{k}(n)- & \sum_{i=1}^{k} c_{k-\imath} g^{k-i}(n) \\
= & \alpha^{k} n+\left(\frac{1}{2}\right) \frac{\alpha^{k}-1}{\alpha-1}-\sum_{\jmath=1}^{k} r, \alpha^{k-\jmath} \\
& -\sum_{i=1}^{k} c_{k-1}\left(\alpha^{k-1} n+\left(\frac{1}{2}\right) \frac{\alpha^{k-1}-1}{\alpha-1}-\sum_{j=1}^{k-i} r, \alpha^{k-\imath-\jmath}\right)
\end{aligned}
$$




$$
\begin{aligned}
= & n f(\alpha)+\frac{1}{2(\alpha-1)}\left(\alpha^{k-1}-c_{k-1}\left(\alpha^{k-1}-1\right)-c_{k-2}\left(\alpha^{k-2}-1\right)-\ldots-c_{1}(\alpha-1)\right) \\
& -r_{1}\left(\alpha^{k-1}-c_{k-1} \alpha^{k-2}-\ldots-c_{2} \alpha-c_{1}\right)-r_{2}\left(\alpha^{k-2}-c_{k-1} \alpha^{k-3}-\ldots-c_{3} \alpha-c_{2}\right) \\
& -\ldots-r_{k-1}\left(\alpha-c_{k-1}\right)-r_{k},
\end{aligned}
$$

which equals $M$. Now $g^{k}(n)-\sum_{\imath=1}^{k} c_{k-\imath} g^{k-\imath}(n)$, as an integral linear combination of integers, is itself an integer. In order for this to be zero, it is necessary and sufficient that $|M|<1$.

CorollaRY 1: If $c_{\imath} \geq 0$, for $0 \leq i \leq k-1$, are integers satisfying

$$
c_{k-1} \geq 1+c_{0}+c_{1}+\ldots+c_{k-2}
$$

then the array $s(i, j)$ is a Stolarsky array.

Proof: Let $f(x)$ be as in (2.2) with $c_{\imath}$ as in (3.2). Since $f(x)>0$ for all $x \geq c_{k-1}+1$, and $f\left(c_{k-1}\right)<0$, the dominant real root $\alpha$ satisfies $c_{k-1}<\alpha \leq c_{k-1}+1$. Then

$$
M<\frac{1}{2\left(c_{k-1}-1\right)}\left(c_{0}+c_{1}+\ldots+c_{k-2}+c_{k-1}-1\right),
$$

since each of the numbers

$$
\frac{r_{1} c_{0}}{\alpha}, \frac{r_{2}\left(c_{0}+c_{1} \alpha\right)}{\alpha^{2}}, \ldots, \frac{r_{k-1}\left(c_{0}+c_{1} \alpha+\ldots+c_{k-2} \alpha^{k-2}\right)}{\alpha^{k-1}}, r_{k}
$$

is nonnegative and $1<c_{k-1}<\alpha$.

Consequently, (3.2) implies

$$
M<\frac{1}{2\left(c_{k-1}-1\right)}\left(2 c_{k-1}-2\right)=1 .
$$

To see that $M>-1$ also, substitute $s_{i}=1-r_{i}$ for $i=1,2, \ldots, k$ to find that

$$
\begin{aligned}
M= & \frac{-1}{2(\alpha-1)}\left(c_{0}+c_{1}+\ldots+c_{k-1}-1\right)+\frac{s_{1} c_{0}}{\alpha}+\frac{s_{2}\left(c_{0}+c_{1} \alpha\right)}{\alpha^{2}} \\
& +\frac{s_{3}\left(c_{0}+c_{1} \alpha+c_{2} \alpha^{2}\right)}{\alpha^{3}}+\ldots+\frac{s_{k-1}\left(c_{0}+c_{1} \alpha+\ldots+c_{k-2} \alpha^{k-2}\right)}{\alpha^{k-1}}+s_{k} .
\end{aligned}
$$

Since all these multiples of the $s_{\mathfrak{z}}$ are nonnegative,

$$
M>-\frac{1}{2(\alpha-1)}\left(c_{0}+c_{1}+\ldots+c_{k-1}-1\right)
$$

so that $M>-1$.

Corollary 1 shows that there exist Stolarsky arrays of every order $k \geq 2$. However, it is possible for $|M|$ to be less than 1 even when inequality (3.2) fails. Corollaries 2 and 3 reveal two such cases.

Corollary 2: Let $\alpha$ be the dominant real root of the polynomial

$$
p(x)=x^{k}-x^{k-1}-x^{k-2}-\ldots-x-1, k \geq 2 .
$$

Then the Stolarsky array $\{s(i, j)\}$ defined in Theorem 1 is a Stolarsky array having characteristic polynomial $(x-1) p(x)$.

Proof: Write

$$
\begin{aligned}
(x-1) p(x) & =(x-1)\left(x^{k}-a_{k-1} x^{k-1}-a_{k-2} x^{k-2}-\ldots-a_{1} x-a_{0}\right) \\
& =x^{k+1}-c_{k} x^{k}-c_{k-1} x^{k-1}-\ldots-c_{1} x-c_{0},
\end{aligned}
$$


where $c_{k}=a_{k-1}+1, c_{k-1}=a_{k-2}-a_{k-1}, \ldots, c_{1}=a_{0}-a_{1}$, and $c_{0}=-a_{0}$.

Then $c_{0}+c_{1}+\ldots+c_{k-1}+c_{k}-1=0$, so that

$$
\begin{aligned}
M & =-\frac{r_{1} c_{0}}{\alpha}-\frac{r_{2}\left(c_{0}+c_{1} \alpha\right)}{\alpha^{2}}-\frac{r_{3}\left(c_{0}+c_{1} \alpha+c_{2} \alpha^{2}\right)}{\alpha^{3}} \\
& -\ldots-\frac{r_{k}\left(c_{0}+c_{1} \alpha+\ldots+c_{k-1} \alpha^{k-1}\right)}{\alpha^{k}}-r_{k+1}
\end{aligned}
$$

For the case at hand, namely, $c_{0}=-1$ and $c_{i}=0$ for $1 \leq i \leq k-1$, we have

$$
M=-r_{k+1}+\sum_{i=1}^{k} \frac{r_{i}}{\alpha^{i}}<\frac{1}{\alpha^{k}} \sum_{i=0}^{k-1} \alpha^{i}=1 .
$$

Also, clearly, $M>-1$. By Theorem $1,\{s(i, j)\}$ is a Stolarsky array.

Corollary 3: Suppose that $p(x)=x^{3}-a_{2} x^{2}-a_{1} x-a_{0}$ has a dominant real root $\alpha$ that satisfies the inequalities

$$
a_{0} \geq 1, a_{1} \geq a_{0}\left(1-\frac{1}{\alpha}\right), \text { and } a_{2} \geq\left(\frac{a_{0}}{\alpha}+a_{1}\right)\left(1-\frac{1}{\alpha}\right) .
$$

Then the array $\{s(i, j)\}$ is a Stolarsky array.

Proof: As in the proof of Corollary 2, we have

$$
M=\frac{r_{1} a_{0}}{\alpha}+\frac{r_{2}\left[a_{0}+\alpha\left(a_{1}-a_{0}\right)\right]}{\alpha^{2}}+\frac{r_{3}\left[a_{0}+\alpha\left(a_{1}-a_{0}\right)+\alpha^{2}\left(a_{2}-a_{1}\right)\right]}{\alpha^{3}}-r_{4}
$$

A sufficient condition that $|M|<1$ is that the coefficients of $r_{1}, r_{2}$, and $r_{3}$ be nonnegative. These three inequalities are easily seen to be equivalent to the three stated in the corollary.

\section{CONCLUDING REMARKS.}

Corollary 3 applies to several cases not previously covered. Following is a table showing several relevant choices of $a_{2}, a_{1}, a_{0}$, the derived coefficients, and the dominant real root $\alpha$. Here, the characteristic polynomial is $(x-1) p(x)$, so that $c_{3}=a_{2}+1, c_{2}=a_{1}-a_{2}, c_{1}=a_{0}-a_{1}$, and $c_{0}=-a_{0}$.

\begin{tabular}{rrr|rrrr|c}
$a_{2}$ & $a_{1}$ & $a_{0}$ & $c_{3}$ & $c_{2}$ & $c_{1}$ & $c_{0}$ & $\alpha$ \\
\hline 1 & 1 & 1 & 2 & 0 & 0 & -1 & 1.83928675521416 \\
2 & 1 & 1 & 3 & -1 & 0 & -1 & 2.54681827688408 \\
2 & 2 & 1 & 3 & 0 & -1 & -1 & 2.83117720720334 \\
3 & 2 & 1 & 4 & -1 & -1 & -1 & 3.62736508471183
\end{tabular}

Table 3: Examples for Corollary 3

For example, to generate a $(2,0,0,-1)$ Stolarsky array, let $\alpha=1.83928675521416$, let $s(i, j)=$ $\left[\alpha j+\frac{1}{2}\right]$ for $j \geq 1$, and define $s(i, j)$ via iteration as stated in Theorem 1 with $s(1,1)=1$. See Table 4.

A notable feature of this array is that the linear recurrence for Row $i$ is given by

$$
s(i, j)=2 s(i, j-1)-s(i, j-4) \text { for } j \geq 5 \text { and } i \geq 1,
$$

and not by

$$
s(i, j)=s(i, j-1)+s(i, j-2)+s(i, j-3),
$$


as might have been expected since $a$ is a root of $x^{3}-x^{2}-x-1$. To see that (4.1) fails, consider the numbers $8,15,28,52$, in Row 4 . Of course, $x^{4}-2 x^{3}+1$ is a multiple of $x^{3}-x^{2}-x-1$. One wonders if there is a Stolarsky array in which at least one row satisfies a second-order recurrence, rows without this property satisfy a third-order recurrence, and the two corresponding characteristic polynomials are relatively prime.

Finally, we note that the arrays $s(i, j)$ investigated in this article have "almost geometric" rows, in the sense that $s(\imath, j+1) / s(\imath, j)$ stays close to $\alpha$. Moreover, they also have "almost arithmetic" columns. Perhaps someone will wish to investigate these properties further.

$\begin{array}{rrrrrrrrrrr}1 & 2 & 4 & 7 & 13 & 24 & 44 & 81 & 149 & 274 & \ldots \\ 3 & 6 & 11 & 20 & 37 & 68 & 125 & 230 & 423 & 778 & \ldots \\ 5 & 9 & 17 & 31 & 57 & 105 & 193 & 355 & 653 & 1201 & \ldots \\ 8 & 15 & 28 & 52 & 96 & 177 & 326 & 600 & 1104 & 2031 & \ldots \\ \vdots & & & & & & & & & & \end{array}$

Table 4: A $(2,0,0,-1)$ Stolarsky Array

\section{REFERENCES}

1. STOLARSKY, K.B. A Set of Generalized Fibonacci Sequences Such That Each Natural Number Belongs to Exactly One, The Fibonacci Quarterly 15 (1977), 224.

2. BURKE, J. R. and BERGUM, G. E. Covering the Integers With Linear Recurrences in Applications of Fibonacci Numbers - Proceedings of the Second International Conference on Fibonacci Numbers and Their Applications, 146-147, Kluwer Academic Publishers, Dordrecht, The Netherlands, 1988.

3. BUTCHER, J. C. On a Conjecture Concerning a Set of Sequences Satisfying The Fibonacci Difference Equation, The Fibonacci Quarterly 16 (1978), 81-83.

4. GBUR, M. E. A Generalization of a Problem of Stolarsky, The Fibonacci Quarterly 19 (1981), $117-121$.

5. HENDY, M. D. Stolarsky's Distribution of the Positive Integers, The Fibonacci Quarterly 16 (1978), 70-80.

6. KIMBERLING, C. H. Second-Order Recurrence and Iterates of $\left[\alpha n+\frac{1}{2}\right]$, to appear in The Fibonacci Quarterly.

7. MORRISON, D. R. A Stolarsky Array of Wythoff Pairs, in A Collection of Manuscripts Related to the Fibonacci Sequence, 134-136, Fibonacci Association, Santa Clara, 1980. 


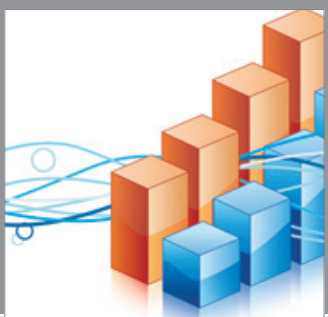

Advances in

Operations Research

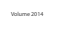

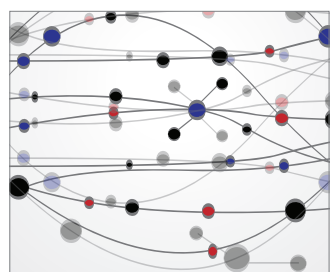

\section{The Scientific} World Journal
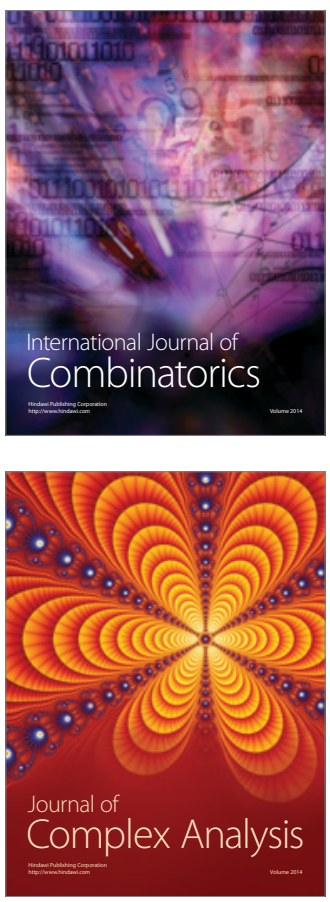

International Journal of

Mathematics and

Mathematical

Sciences
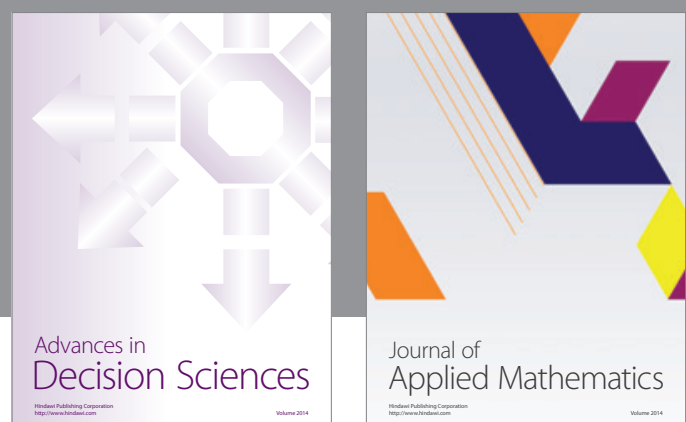

Journal of

Applied Mathematics
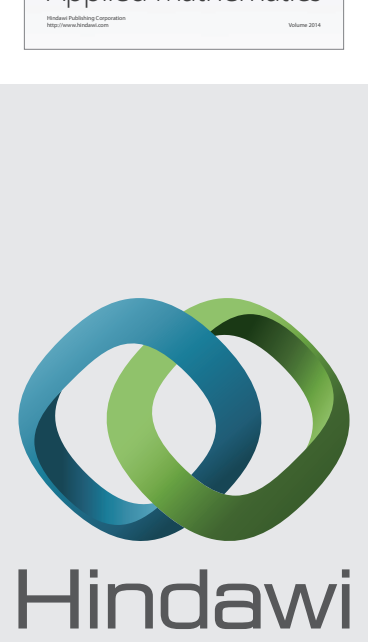

Submit your manuscripts at http://www.hindawi.com
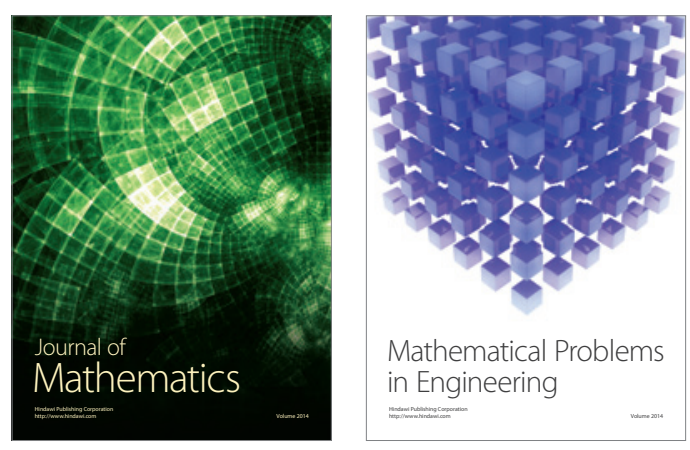

Mathematical Problems in Engineering
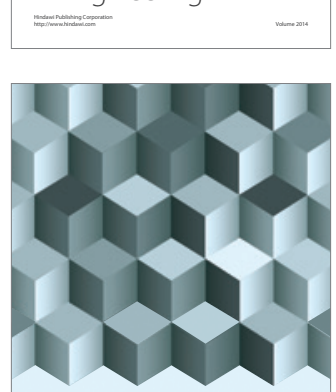

Journal of

Function Spaces
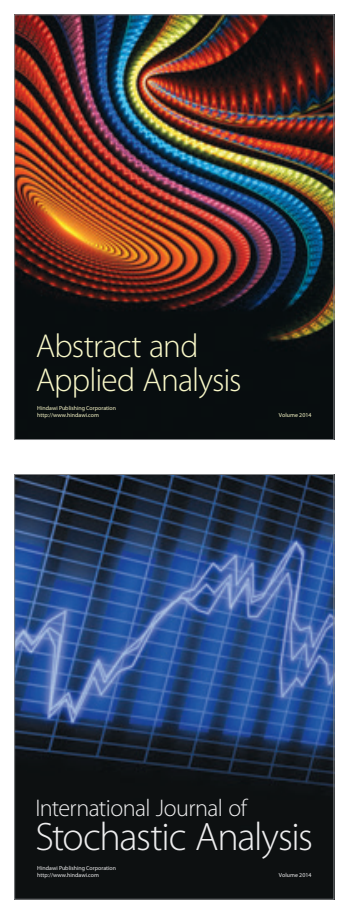

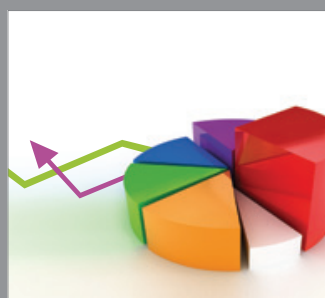

ournal of

Probability and Statistics

Promensencen
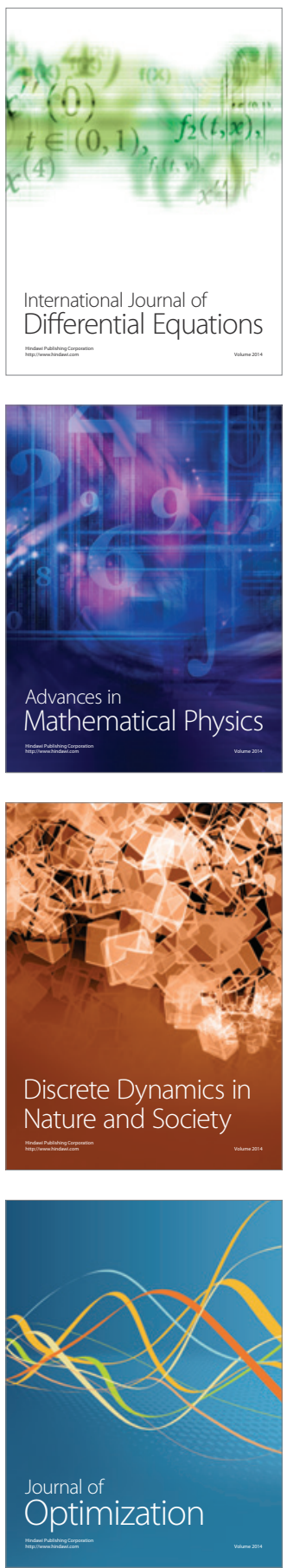